\title{
6. STREITGESPRÄCHE ÜBER DAS HEILSGESCHEHEN UND SATANSPROZESSE
}

Einige Texte im untersuchten Corpus inszenieren eine Diskussion über das Heilsgeschehen zwischen einem Vertreter der Hölle und einem Vertreter des Himmels. Der Kontrast der Figuren und ihrer Argumentationen charakterisiert die Werke als Streitgespräche, doch unterscheiden sie sich von den restlichen Streitgesprächen der Zeit vor allem durch den fehlenden Bezug auf zeitgenössische Polemiken mit gesellschaftlicher Breitenwirkung. Es handelt sich um folgende Werke: Conflictus inter Deum et diabolum (R2), Quaestio Christi ad Cain (R3), Processus Satanae (in drei Fassungen: R5a, R5b, R5c), Argumenta quae fecit diabolus Christo pendenti in cruce (R7a), Quaestiones quae fuerunt inter Christum et diabolum (R7b), Dialogus lucis et tenebrarum (R19). Da jeder Text eine andere Werksbezeichnung im Titel trägt, werden sie ab hier nur mit dem ersten Titelwort benannt. Processus und Dialogus unterscheiden sich von den Streitgesprächen dadurch, dass sie eine sorgfältig ausgearbeitete Rahmenhandlung aufweisen und die Debatte im Rahmen eines Prozesses stattfinden lassen.

Dies ist eine bisher wenig bekannte und kaum erforschte Gruppe, daher sind grundlegende Fragen noch offen: die Identität der Verfasser, die Entstehungszeit der Werke und die Beziehungen zwischen den verschiedenen Texten und Textstufen. Was die Autorschaft der Texte angeht, bietet die Überlieferung nur für zwei Fassungen des Processus Autornamen, und zwar Guido de Collemedio für die Advocacia-Fassung und Bartolus de Saxoferrato für die Bartolo-Fassung, aber ihre Autorschaft ist keineswegs gesichert. Die restlichen Werke sind anonym überliefert und es gibt keine Anhaltspunkte zur Bestimmung des Autors. Hinzu kommt, dass die ganze Gruppe schwer datierbar ist. Nur für den Dialogus lässt sich ein sicherer Entstehungszeitraum bestimmen; er erstreckt sich vom zweiten Viertel des 13. bis zum Anfang des 14. Jahrhunderts. Die restlichen Texte können aus inhaltlichen Gründen frühestens um die Mitte des 12. Jahrhunderts entstanden sein, der Terminus ante quem wird durch die Überlieferung oder durch volkssprachliche Fassungen gegeben und variiert zwischen dem 13. (Conflictus) und dem 15. Jahrhundert (Argumenta). Weitere offene Fragen bestehen in Bezug auf die drei Fassungen des Processus sowie auf die 
engverwandten Quaestiones und Argumenta. Es ist unklar, in welcher Reihenfolge die Fassungen entstanden sind und welche Beziehungen zu einigen volkssprachlichen, ebenfalls bisher wenig untersuchten Texten bestehen. Auch lassen sich Bezüge zwischen den verschiedenen Werken dieser Gruppe nicht feststellen.

Die Gesprächskonstellation ist in der ganzen Textgruppe gleich: Ein Vertreter der Hölle beanstandet, dass Jesus mit seinem Tod dem Teufel sein legitimes Eigentum, nämlich die Menschheit, entrissen habe und beansprucht gleichzeitig die Erlösung der Höllenbewohner; ein Himmelsvertreter erklärt die Notwendigkeit und unumkehrbare Wirkung der Menschwerdung Gottes und führt an, warum die Strafe des Teufels nicht aufgehoben werden könne. Die Personen variieren: In Argumenta, Quaestiones und Conflictus treten Jesus und der Teufel als Streitende auf, in der Quaestio diskutieren Jesus und Kain, der hier der Fürsprecher der Hölle ist. Im Dialogus wird Jesus in der Auseinandersetzung mit dem Teufel durch Gabriel vertreten. In den Processus klagt ein Teufel als Anwalt der Hölle nicht Jesus, sondern die Menschheit an, die von Maria als Anwältin vertreten wird.

Der Kontext, in dem das Gespräch stattfindet, variiert. In Argumenta und Quaestiones streitet ein Teufel mit dem gekreuzigten Jesus. Alle anderen Werke lassen die Diskussion nach Jesu Tod und seiner Höllenfahrt stattfinden: in der Quaestio findet das Streitgespräch in der Hölle statt, als Kain merkt, dass er nicht zusammen mit den Altvätern erlöst wird; im Conflictus, in den Processus und im Dialogus ereignet sich die Auseinandersetzung im Himmel, wo der Teufel oder sein Vertreter vor Gott erscheinen, um gegen die Erstürmung der Hölle zu protestieren. Die Höllenfahrt, der Aufbruch der Tore, die späte Erkenntnis Satans, dass er Gottes Sohn getötet und damit sein Recht auf die Menschheit verwirkt hat, und die Befreiung der Altväter werden zwar nicht geschildert, sie bilden aber den bekannten Hintergrund. Der Glaube an Christi Höllenfahrt (Descensus ad inferos) beruht nicht auf den kanonischen Schriften, obwohl er im apostolischen Glaubensbekenntnis verankert ist. Die bekannteste Erzählung der Geschehnisse in der Hölle bietet eine im Mittelalter weitverbreitete und vielgelesene apokryphe Schrift, das Evangelium Nicodemi ${ }^{1}$. Die italienische Fassung von Argumenta

${ }^{1}$ Izydorczyk, Manuscripts, konnte 437 mittelalterliche Handschriften des Evangelium Nicodemi ausfindig machen. Zur Rezeption in der mittelalterlichen Literatur vgl. die in Izydorczyk, The Medieval Gospel, versammelten Aufsätze. 
und Quaestiones, der Piaito ch'ebbe Dio con l'inimico hat ebenfalls den Descensus als Hintergrund. In einer kurzen Eingangserzählung wird geschildert, wie der Teufel sich über die Kreuzigung freut, sich aber von der Aufregung der Altväter irritiert fühlt. Als Jesus in die Hölle eindringt und die Gerechten aus der Vorhölle befreit, versteht er, was wirklich geschehen ist, und erscheint im Himmel, um gegen Christi Vorgehen zu protestieren. Dort findet das Gespräch statt. In den beiden lateinischen Fassungen hingegen (von denen nicht klar ist, ob sie Vorlage oder Übersetzung des Piaito sind) diskutiert der Teufel mit dem Gekreuzigten. Der Redaktor oder Schreiber der Argumenta äußert sogar im Kolophon eine gewisse Skepsis gegenüber seiner Vorlage, weil der Teufel im gekreuzigten Christus den Heiland erkennt, während er Jesu wahre Identität erst nach Erstürmung der Hölle erfahren haben soll ${ }^{2}$.

Der Descensus ad inferos wird in der lateinischen Literatur in verschiedenen Nacherzählungen des Evangelium Nicodemi sowie in Hymnen von Prudentius, Beda und Audradus geschildert. Im Spätmittelalter kommt der Descensus vor allem in erbaulichen Schriften mit Darstellungen der Heilsgeschichte vor, wie im Speculum historiale Vinzenz' von Beauvais oder in der Legenda aurea Jakobs von Voragine ${ }^{3}$. Im theologischen Fachschrifttum wurden seit der Kirchenväterzeit verschiedene Fragen in diesem Zusammenhang diskutiert, zum Beispiel, was Jesus in der Hölle machte und wen er befreite ${ }^{4}$. In dieser breiten Rezeption des Evangelium Nicodemi in der lateinischen Literatur fehlen aber Auseinandersetzungen zwischen Jesus und dem Teufel, die als Vorbilder für die Streitgespräche in Frage kämen.

Die direkte Konfrontation zwischen Gott und seinem Widersacher findet sich in verschiedenen literarischen Traditionen, ohne dass es möglich wäre, Querbezüge festzustellen. In der byzantinischen Literatur gibt es ein Streitgespräch zwischen Christus und dem Teufel, das ins Altslawische übersetzt wurde; aber sowohl die narrative Umrahmung

\footnotetext{
${ }^{2}$ Predicta, licet sint verisimiliter fulcita veritate, tamen, quia ex nulla certa scriptura probantur pro ficticiis habentur, cum praesertim quod demonem latuit Christum esse verum Deum usque ad descensum inferni et eius fractionem scilicet limbi, quod vocat propheta in verbis istis potestativis Tollite portas etc. (Hs. Vaticano, Vat. lat. 10499, fol. 39r).

${ }^{3} \mathrm{Zu}$ den lateinischen Texten vgl. Izydorczyk, „The Evangelium“, der allerdings weder die Hymnen noch die hier besprochenen Streitgespräche und Satansprozesse erwähnt. Zu den Hymnen vgl. Campbell, ,To Hell and back“, 125-129.

${ }^{4}$ Zur theologischen Diskussion in der Väterzeit und im Mittelalter vgl. Turner, „Descendit ad inferos“; Campbell, „To Hell and back“, 118-125; Maas, Gott und die Hölle, 133-164.
} 
als auch die behandelten Themen unterscheiden es von den lateinischen Dialogen $^{5}$. Ebenfalls keine Beziehungen zu den lateinischen Texten haben drei syrische Streitgedichte, in denen der Teufel am Kreuz den zweifelnden Tod dazu überredet, den Gekreuzigten mitzunehmen. Nach der Auferstehung beklagt sich der Tod, der Teufel und er seien nun besiegt ${ }^{6}$. In den volkssprachlichen Literaturen finden sich etliche Texte, die sich mit den hier untersuchten inhaltlich und formell berühren; sie sind jedoch zeitgleich mit den lateinischen Werken und stellen zum Teil deren Übersetzungen dar?

Anregung für die hier besprochenen Streitgespräche dürften eher einige Szenen gegeben haben, die in der Literatur des 12. Jahrhunderts häufig vorkommen: die innertrinitarische Beratung und der Streit der Töchter Gottes. In ersterer beraten sich die Personen der Dreifaltigkeit über die Schöpfung des Menschen oder über seine Erlösung. In der zweiten, die von Psalm 84,11 ausgeht, streiten Veritas und Justitia einerseits gegen Misericordia und Pax andererseits über die Erlösung der Menschheit vor Gott als Richter. Diese Diskussionen finden sich innerhalb von längeren Texten, in Predigten, Bibelkommentaren, theologischen Abhandlungen oder dramatischen Darstellungen der

${ }^{5}$ Vgl. Casey/Thomson. In den byzantinischen Gesprächen sucht der Teufel Jesus auf einem Berg beim Fasten heim. Er fordert Jesus dazu auf, in den Himmel zurückzugehen und ihm und seinen Verbündeten die Welt zu überlassen. Jesus antwortet, er sei gekommen, um die Menschheit zu retten, worauf sich ein Gespräch darüber anschließt, wer gerettet werden soll und was mit den Verdammten geschehen werde. Zuletzt greift der Teufel mit seinen Truppen Jesus an, dieser wird aber verklärt und schlägt ihn in die Flucht. Der Text endet mit einer Schilderung der letzten Dinge. Es gibt Abweichungen zwischen den zwei erhaltenen griechischen Handschriften und den zwei Handschriften der slawischen Übersetzung. Letzere sind aus dem 15. Jahrhundert, die griechischen Textzeugen aus dem Spätmittelalter; die Editoren Casey und Thomson halten es dennoch für denkbar, dass der Text einen spätantiken Ursprung haben könnte.

${ }^{6}$ Vgl. Reinink, „Ein syrisches Streitgespräch“. Eines der Gedichte ist von Ephraem Syrus.

${ }_{7}$ Zu den volkssprachlichen Fassungen von Argumenta, Quaestiones, Processus und Dialogus siehe die jeweiligen Repertoriumseinträge. Eine Übersicht über die Satansprozesse in Latein und den Volkssprachen bei Cardelle de Hartmann, „Die Processus“, 417-419. Ein weiteres Streitgespräch zwischen Christus und Lucifer bildet den Kern eines englischen Gedichtes über die Erstürmung der Hölle mit dem Titel Devils' Parliament (15. Jahrhundert), vgl. Marx, „The Gospel“, 245-247. Marx, der den Conflictus ediert hat, findet beide Texte ähnlich, wenn er auch keine Abhängigkeit feststellen kann. Ansonsten sind in den ausführlichen Untersuchungen zur Rezeption des Evangelium Nicodemi in der Aufsatzsammlung Izydorczyk, The Medieval Gospel, kaum Hinweise auf die Streitgespräche zur Heilsgeschichte zu finden. Nur der Piaito wird besprochen, vgl. Ianucci, ,The Gospel“, 187-188. 
Heilsgeschichte ${ }^{8}$. Thematisch stehen sie den Streitgesprächen zum Heilsgeschehen nahe, aber sie lassen andere Figuren auftreten ${ }^{9}$. Die größte Ähnlichkeit mit den spätmittelalterlichen Dialogen zwischen Himmelund Höllenvertretern weist ein kurzer Text Hugos von St. Victor auf. Unter seinen Adnotationes elucidatoriae in quosdam Psalmos David findet sich ein Kommentar zum Psalm 15,6 $6^{10}$, in dem eine Auseinandersetzung zwischen Gott und dem Teufel in indirekter Rede geschildert wird. Gott äußert seinen Willen, die Menschheit zu retten, und der Teufel will ihn davon abbringen. Da ihm dies nicht glückt, überzeugt er Gott, sich die Menschheit mit ihm zu teilen.

Innerhalb der lateinischen Streitgespräche lassen sich nach der Ausarbeitung der Rahmenhandlung und der Charakterisierung der Personen zwei Gruppen unterscheiden. Quaestiones, Argumenta, Quaestio und Conflictus nennen oder schildern nur kurz die Umstände des Gesprächs. Dieses verläuft so, dass der Höllenvertreter Argumente vorbringt, die von Jesus erwidert werden. Der Conflictus hat außerdem eine Richterfigur: die Engel (troni) fällen nach der Auseinandersetzung das Urteil, dass die Klage des Teufels abgewiesen werden soll. Der Processus in seinen drei Fassungen und der Dialogus zeichnen sich hingegen dadurch aus, dass sie die Handlung ausarbeiten und zu diesem Zweck mehrere Personen auftreten lassen. Beide Werke gestalten das Gespräch als eine Gerichtsverhandlung: ein Teufel erscheint vor dem himmlischen Gericht und verklagt die Menschheit, die sich seiner Macht entzogen (Processus), beziehungsweise Jesus, der ihm sein Eigentum entwendet habe (Dialogus). Während daraufhin im Conflictus, der am Anfang dieselbe Situation - wenn auch wesentlich knapper - skizziert, die Reden von Teufel und Jesus und die Abweisung der Klage durch die Troni folgen, findet in den anderen Texten der Prozess wirklich statt und wird genau ausgearbeitet. In beiden Werken findet sich ein Himmelsbewohner als Anwalt für den Angeklagten (Maria vertritt die Menschheit im Processus, Gabriel ist Jesu Vertreter im Dialogus) und ein lebhaftes Streitgespräch schließt sich an. Ankläger und Verteidiger argumentieren für die jeweilige Position und versuchen, die gegnerischen Argumente zu entkräften;

\footnotetext{
8 Vgl. Ohly, „Die Trinität“; siehe auch unten 242-244.

9 Diese thematische Übereinstimmung hat zur Aufnahme vom Streit der Töchter Gottes in einen Satansprozess geführt. Die Mascharon-Fassung des Processus lässt Maria und Mascharon weitere Anwälte zur Unterstützung heranziehen. Misericordia und Pax kommen Maria zu Hilfe, Veritas und Justitia unterstützen Mascharon.

${ }_{10}$ PL 177, 596-597.
} 
sie zögern nicht, den Widersacher zu beleidigen und zu diskreditieren. Der Richter schaltet sich wiederholt ein, um für Ruhe zu sorgen oder weil ein Zwischenurteil beantragt wurde, und fällt am Ende ein Urteil. Die Gerichtsverhandlung findet vor einem Publikum statt, das je nach Situation wehklagt oder mitjubelt und von den Anwälten angesprochen wird. In beiden Texten wird das Gespräch immer wieder von narrativen Einschüben unterbrochen, die über die Reaktionen und Bewegungen der beteiligten Personen berichten. Insgesamt weichen Processus und Dialogus sehr stark von den anderen Streitgesprächen ab: die Figuren werden durch ihre Worte und Taten genauer charakterisiert, ihr Gespräch wird durch das Ineinandergreifen der Argumente organischer, die situativen Bezüge und die persönlichen Ansprachen sorgen für einen dramatischen Ton, die narrativen Einschübe verwischen an manchen Stellen die Gesprächsform. Auch in der Art der Argumentation unterscheiden sich Processus und Dialogus von den anderen Streitgesprächen. In Quaestiones, Argumenta, Quaestio und Conflictus finden sich vor allem theologische Inhalte, gelegentlich auch juristische Argumentation, die jedoch nicht sehr ausgearbeitet ist. Processus und Dialogus hingegen gestalten den Prozess sorgfältig nach dem Verfahren des gelehrten Rechts, die Argumentation ist in der Hauptsache juristisch, es fehlen auch nicht die Hinweise auf die genauen Quellentexte aus dem kanonischen wie aus dem Zivilrecht.

Die Unterscheidung zwischen Streitgesprächen über das Heilsgeschehen und Satansprozessen im Titel dieses Abschnitts will diesen Unterschieden gerecht werden. Die Erzählanteile in Processus und Dialogus, die Anzahl der auftretenden Personen, das Gewicht der Fiktion erlauben nicht, diese Texte uneingeschränkt als Streitgespräche zu identifizieren. „Satansprozesse“ wiederum ist eine Bezeichnung, die sich lediglich auf den Inhalt bezieht. Die Satansprozesse sind Werke, die den vom Teufel im Himmel angestrengten Prozess um die Wiedererlangung der Menschheit inszenieren, formell gehören sie verschiedenen Textsorten $\mathrm{an}^{11}$. Processus und Dialogus lassen sich schwer definieren, sie bilden eine Mischform, die Elemente der erzählenden, der dramatischen und der didaktischen Literatur vereint. Die Übersetzungen und Bearbeitungen in den Volkssprachen spiegeln diese Unbestimmtheit wider. Die Processus-Fassungen wurden als erzählende Gedichte (Advocacie Nostre Dame,

${ }^{11} \mathrm{Zu}$ den Satansprozessen vgl. Strothmann, Die Gerichtsverhandlung, 32-45; Cardelle de Hartmann, „Die Processus“, „Satan“. 
Maskeroen-Episode in Jakobs van Maerlant Boek van Merline) oder als Dramen (Mystère de l'Advocacie, Aucto de acusación) übertragen. Die deutschen Übersetzer der Bartolo-Fassung haben eher ihren didaktischen Wert (Vermittlung des gelehrten Rechts) gesehen: Georg Alt pries den Text im Titel als nützlicher gerichteshandel, Ulrich Tenngler integrierte ihn in seinen Layen Spiegel, der Laien in den Städten mit dem gelehrten Recht im Hinblick auf ihre Tätigkeit als Schöffen - vertraut machen wollte. Am weitesten auf diesem Weg der Wissensvermittlung ging Jacobus von Theramo in seinem Belial ${ }^{2}$, in dem der Prozess minutiös nach den Regeln des Verfahrensrechts, unter Beachtung der vorgeschriebenen Verteilung auf schriftliche und mündliche Verhandlung und Aufnahme aller benötigten Urkunden aufgerollt wird. Im Belial beschränkt sich die Personensprache auf die mündlichen Partien des Verfahrens, die Ähnlichkeit mit den Streitgesprächen verschwindet fast vollständig.

Die mittelalterliche Titelgebung ist uneinheitlich. Von den zwei für Streitgespräche charakteristischen Titeln, dialogus und disputatio, kommt in der ersten Gruppe dialogus nur in zwei Handschriften der Quaestio, disputatio nirgends vor. Die Werke tragen jedoch Titel, die auf eine Diskussion hinweisen: Conflictus und Altercatio (in einer Handschrift des Conflictus) sind zwei für Streitgedichte kennzeichnende Titel; Quaestio, Quaestiones und sogar Argumenta weisen eher auf die Praxis der scholastischen Disputation hin. Bei den Processus findet sich hingegen keine Andeutung auf die Debatte innerhalb des Textes. Der Titel variiert sehr stark, neben Processus finden sich die Bezeichnungen tractatus und libellus, die auf keinen bestimmten Texttyp deuten. Überraschenderweise heißt der andere Satansprozess in der Lilienfelder Handschrift Dialogus lucis et tenebrarum. Der Schreiber der Kasseler Handschrift, die eine Abschrift der Lilienfelder sein dürfte ${ }^{13}$, fand wohl diesen Titel unpassend und ersetzte ihn durch Ordo iudicii inter Christum et Sathanam.

Die Untersuchung Otts ${ }^{14}$ zur Rezeption des Belial und seiner deutschen Übersetzung klärte einige Aspekte in Bezug auf das Publikum dieser Texte. Ott konnte feststellen, dass sowohl die lateinische als auch die deutsche Fassung im Überlieferungskontext theologischer und juristischer Schriften vorkommen. Dabei weisen die mit dem lateinischen Belial überlieferten Texte eher auf einen theologisch-kanonistisch

${ }^{12} \mathrm{Zu}$ diesem Autor vgl. Ott, „Jacobus“ und A17. Zum Belial vgl. Ott, Rechtspraxis.

${ }^{13}$ Dies zeigt eine erste, von mir durchgeführte Kollationierung der Handschriften.

${ }^{14} \mathrm{Ott}$, Rechtspraxis. 
ausgebildeten Rezipientenkreis, die Mitüberlieferung der deutschen Übersetzung auf die Verwendung durch juristische Laienpraktiker hin. Bei den früheren, hier untersuchten Texten lassen sich zur Rezeption wegen der dürftigen Erschließungslage nur einige Beobachtungen mitteilen, die von einer eingehenden Untersuchung der Überlieferung bestätigt oder korrigiert werden müssten. Die Schriften werden in der Regel mit theologischen, gelegentlich mit juristischen Schriften oder in Codices, die theologische und juristische Schriften vereinen, überliefert ${ }^{15}$. Es lässt sich dabei feststellen, dass die Texte mit geringerem Gewicht der juristischen Argumentation (Conflictus, Quaestio, Argumenta, Quaestiones) vorwiegend zusammen mit theologischen Schriften überliefert werden. Eine Ausnahme ist wohl die Erfurter Handschrift der Quaestio: sie enthält Texte, die sich für Lektüre und Kommentar im (fortgeschrittenen) Grammatik- und Rhetorik-Unterricht eignen (Persius, Ovid, Petrarcas Griseldis, De planctu naturae des Alain de Lille). Vor allem die ProcessusFassungen finden sich zusammen mit juristischen Schriften. Nicht nur deshalb gewinnt man den Eindruck, dass die juristischen Bestandteile des Textes auf Interesse stießen: In den Handschriften München, BSB, Clm 27421 und Clm 3331 wurden die genauen Belege für die juristischen Argumente an den Rand geschrieben, in der Bartolo-Fassung wurden sie durchgängig in den Text eingefügt. Wenn man die Provenienz der Codices in Augenschein nimmt, lässt sich für die ganze Gruppe feststellen, dass einige im Umkreis der Universitäten entstanden ${ }^{16}$, während andere Klöstern gehörten. Bei zwei Handschriften lässt sich sogar nachweisen, dass sie sich im Besitz eines Universitätsdozenten befanden: Oxford, BodL, Bodley 52, die den Conflictus mit theologischen Schriften (davon etliche im Zusammenhang mit dem Armutsstreit und der Polemik gegen die Mendikanten) überliefert, wurde von John Maynsforth, Fellow des Merton College, geschrieben; Lübeck, Cod. theol. lat. 64, die die Quaestio enthält und in der Schreiberwerkstatt des Johannes Fogel geschrieben wurde, gehörte einem Hochschullehrer, nämlich Simon Batz

${ }^{15}$ Folgende sind Beispiele aus der Überlieferung der Processus-Fassungen. Mit juristischen Traktaten: Frankfurt am Main, StUB, Ms. Praed. 61; München, BSB, Clm 27421 (kanonistische Schriften); Leipzig, UB, Haen. 15 (mit Traktaten des Bartolus de Saxoferrato). Mit theologischen Schriften: München, BSB, Clm 3334; Paris, BnF, lat. 18216; Mainz, StB, I, 151; Marseille, BM, 211. Mit theologischen und juristischen Schriften: München, BSB, Cgm 660; Uppsala, UB, C 131; Würzburg, UB, M.p.th.f. 55.

${ }_{16}$ Obwohl die Provenienz dieser Handschriften nicht untersucht wurde und sich deshalb nichts Sicheres sagen lässt, ist es auffällig, dass sich etliche Codices im Besitz von alten Universitäten befinden: Bologna, Paris, Oxford, Leipzig, Erfurt. 
von Homburg, Rektor der Universität Erfurt und später Syndikus der Stadt Lübeck ${ }^{17}$. Etliche Codices waren im Besitz von Klöstern. ProcessusFassungen waren vorhanden z.B. in den Benediktinerklöstern Prüfening (Clm 27421), St. Emmeram (Clm 14831) und Attel (Clm 3334), im Dominikanerkonvent von Frankfurt (Frankfurt, StUB, Mss. Praed. 59, 61, 125) sowie im Konvent der Augustinereremiten in Seemannshausen (Cgm 660). Dies dürfte auf das Universitätsstudium von Mönchen zurückzuführen sein, das im 15. Jahrhundert, der Entstehungszeit all dieser Handschriften, bereits weitverbreitet war $^{18}$. Überraschender ist die Provenienz der Dillinger Handschrift der Quaestio aus der Erfurter Kartause. Hingegen erzählt der Wiener Codex des Dialogus eine vielleicht repräsentative Geschichte. Dieser Codex wurde Anfang des 14. Jahrhunderts von Christian von Lilienfeld geschrieben. Die Zisterze Lilienfeld stand zu dieser Zeit in enger Verbindung mit der Zisterze Heiligenkreuz, wo einige Jahre vorher (wohl zwischen 1295 und 1300) der Mönch Gutolf ein Handbuch des kanonischen Rechts mit dem Titel De ordine iudiciario ${ }^{19}$ in der erklärten Absicht verfasst hatte, seine Mitbrüder für dieses Wissensgebiet zu interessieren. Möglicherweise verfolgte Christian ein ähnliches Ziel. Insgesamt scheinen diese Texte im Umfeld der Universitäten kursiert und ein Publikum interessiert zu haben, das Kenntnisse der Theologie und des Rechts besaß. Vielleicht bildeten die Universitäten das Umfeld, in dem die verschiedenen Autoren mit dieser Darstellungsform von Problemen und Argumenten in Bezug auf die Erlösungsgeschichte in Berührung kamen. Zwar ist es nicht möglich, direkte Abhängigkeiten der Texte untereinander nachzuweisen, aber eine völlig unabhängige Entstehung der Werke ohne gegenseitige Kenntnis scheint kaum vorstellbar. Das juristische Interesse hat wohl im lateinisch lesenden Publikum überwogen, was zu einer juristisch immer genaueren Ausarbeitung geführt hat, bis hin zum Prozessbuch Belial. In Deutschland hat die Teilnahme von juristischen Laienpraktikern an der Rechtspflege Übersetzungen entstehen lassen, die die juristischen Aspekte der Texte herausstellen. In den anderen Volkssprachen überwiegen hingegen die religiösen Aspekte, wobei vor allem eine gefühlvolle Marienfrömmigkeit zum Ausdruck kommt.

17 Mitteilung der Lübecker Bibliothekarin Frau Lukow-Wilms in E-Mail an die Verfasserin (08. Dezember 2005).

${ }_{18}$ Vgl. Vandenbroucke, Pour l'histoire, 14-15.

19 Von Schönbach ediert, Über Gutolf, 59-81. Zu dieser Schrift vgl. Stelzer, Gelehrtes Recht, 136-140. Eine Abschrift befindet sich noch in Lilienfeld: StiftsB, 137, fol. 168v-172r. 